\title{
AN ISOMORPHISM THEOREM FOR FINITELY ADDITIVE MEASURES
}

\author{
DONALD G. AUSTIN
}

A problem which is appealing to the intuition in view of the relative frequency interpretation of probability is to define a measure on a countable space which assigns to each point the measure 0 . Such a measure of course becomes trivial if it is countably additive. Finitely additive measures of this type have been discussed by R. C. Buck [1] and by E. F. Buck and R. C. Buck [2]. In a discussion of the density of sets of integers, R. C. Buck introduces in [1] a special finitely additive measurable space, $\mathcal{D}_{0}^{*}$, containing the arithmetic progressions and assigning to each a measure $m^{*}$ equal to the reciprocal of their period. Special properties of this measure are developed there largely from the number theoretic point of view. In [2] the authors showed that any separable, non atomic, normalized, finitely additive measure was isomorphic to a contraction of $\left[\mathcal{D}_{0}^{*}, m^{*}\right]$.

Necessary and sufficient conditions for a separable, non atomic measure to be point and set isomorphic to the Borel sets have been established by Halmos and von Neumann [3]. Here we consider the analogous problem for a finitely additive measure on a countable space. We show that a wide class of finitely additive measures in a countable space are set isomorphic to Jordan content on $[0,1]$ and point isomorphic to a restriction of Jordan content to a countable dense subset of $[0,1]$.

Let $X$ be a countable space and let $\mu_{P}$ be a real, finitely additive function defined on a countable class $P$ of subsets of $X$. We assume the following properties of $P$ and $\mu_{P}$ :

(i) $E_{1}, E_{2} \in \mathcal{P}$ implies $E_{1} \cap E_{2} \in \mathcal{P}$,

(ii) $E_{1}, E_{2} \in \mathcal{P}, \quad E_{1} \subset E_{2}$ implies there exists $C_{i}$ such that $E_{1}=C_{1} \subset C_{2} \subset \cdots \subset C_{n}=E_{2}$ and $C_{i}-C_{i-1} \in P$,

(iii) $X$ and 0 are in $P$ and $\mu_{P}(X)=1, \mu_{P}(0)=0$, further $E_{1} \in P$, $E_{1} \neq 0$ implies that $\mu_{P}\left(E_{1}\right)>0$.

(iv) For any $s \in X$ there exist $E_{i} \in P$ such that $s \in E_{i}$ and

$$
\lim _{i} \mu_{P} E_{i}=0 \text {. }
$$

The sets $P$ then form a semi-ring (cf. [4, p. 22]), and there is a unique minimal ring $R(\Phi)$ containing $P$ with a finitely additive measure $\mu_{R}$ such that $\mu_{R} E=\mu_{P} E$ if $E \in P$. Indeed it is easy to show

Received by the editors April 12, 1954 and, in revised form, August 5, 1954. 
that $R(P)$ consists of just those sets $E$ which are finite, disjoint unions of sets $E_{1} \cdots E_{n}$ in $P$ and $\mu_{R} E=\sum \mu_{P} E_{i}$. We consider measures $[\mathscr{X}, \mu]$ which are the finite Carathéodory closures of such a collection $\left[R(P), \mu_{R}\right]$ (i.e. $E$ is in $\mathcal{X}$ if and only if, for any $\epsilon>0$ there are sets $G$ and $H$ in $R(P)$ such that $G \supset E, H \supset X-E$ and $\mu_{R}(G \cap H)<\epsilon$; in which case we define $\left.\mu E=\operatorname{glb}\left[\mu_{R} G ; G \in R(P), G \supset E\right]\right)$. We shall refer to the measure spaces $[\mathcal{H}, \mu]$ as $\mathrm{f}$-a measures. It may be remarked that the class $\mathcal{D}_{0}^{*}$ may be defined this way by taking the arithmetic progressions as the semi-ring $\mathcal{P}$. As a second example it may be noted that a finitely additive measure can be defined on a countable, dense set $D$ on the interval $[0,1)$ by defining a set $E$ to be measurable if its closure $\mathrm{Cl} E$ is in the class $\mathcal{F}$ of sets possessing Jordan content and letting the measure of the set, $\nu E$, be the content $m \mathrm{Cl} E$ of its closure. Such a measure arises from the above construction if we let $P$ be the collection of all sets which are the intersection of $D$ with half open rational sub-intervals of $[0,1)$. We show that such a measure space $[\mathcal{C}, \nu]$ is universal in the sense that if $[\mathcal{H}, \mu]$ is any $\mathrm{f}$-a measure, then there is a 1-1 transformation $T$ such that $T(X)$ is a dense set $D$ on $[0,1)$ and $\mu E=\nu T(E)$ when $E$ is in $\mathcal{X}$.

ThEOREM 1. For any $f$-a measure $[\mathcal{H}, \mu]$ there exists a 1-1 transformation $T$ carrying $X$ into a dense set $D$ on $[0,1)$ and satisfying the above condition. Furthermore for any $x \in X$ the numerical value of $T(x)$ can be determined by a finite number of arithmetic operations.

Let $\left\{x_{i}\right\},\left\{E_{i}\right\}$ be orderings of the points of $X$ and the sets of $P$. We shall describe by induction a sub-ordering $E_{i}^{(j)}$ of $P$ (possibly containing some sets more than once) such that for each $i, E_{i}^{(j)}$ is a finite union of sets exhausting $X$ and containing a sub-union equal to $E_{i}$ and such that each set $E_{i}^{(j)}$ is a subset of some set $E_{i-1}^{(k)}$. Let $E_{0}^{1}=X$ and suppose sets $E_{i}^{(j)}, i=0,1,2, \cdots, k-1, j=1,2, \cdots, n_{i}$, have been described; we now determine the sets $E_{\mathbf{k}}^{(j)}, j=1,2, \cdots, n_{k}$. Let $E_{\mathbf{k}}^{(1)}=E_{k} \cap E_{\mathbf{k}-1}^{(1)}$ if this set is not null; in which case by (ii) above there exist sets $E_{k}^{(j)}\left(j=2, \cdots, n_{1}\right)$ such that $E_{k}^{(1)} \cap E_{k}^{(j)}=0(i \neq j)$, $E_{k}^{(j)} \subset E_{k-1}^{(1)}, \quad \bigcup_{j=1}^{n_{1}} \quad E_{\mathbf{k}}^{(j)}=E_{k-1}^{(1)}$, and $E_{\mathbf{k}}^{(j)} \in \mathcal{P}$. If $E_{k} \cap E_{\mathbf{k}-1}^{(1)}=0$ we let $E_{k}^{(1)}=E_{k-1}^{(1)}$. We define a partition of each of the sets $E_{k-1}^{(j)}$ similarly and the resulting sets $E_{\boldsymbol{k}}^{(f)}$ have the desired properties. By finite additivity of the function $\mu$ we see that if $E_{k}^{\left(j_{i}\right)}\left(i=1,2, \cdots, n_{j}\right)$ are the subsets of $E_{k}^{(t)}$ contained in $E_{k-1}^{(j)}$, then $\sum_{i_{i=1}}^{n_{j}} \mu E_{k}^{j_{i}}=\mu E_{k-1}^{(j)}$. We shall denote by $x_{i}^{(j)}$ the point $x_{i^{\prime}}$ where $i^{\prime}$ is the minimum $n$ such that $x_{n}$ is in $E_{\mathbf{r}}^{(j)}$. Set transformations $T_{k}$ can be defined on $E_{\mathbf{k}}^{(j)}$ such that $T_{k}\left(E_{i}^{(\Re)}\right)$ is an interval (closed on the left, open on the right) on $[0,1)$ for $k \geqq i$ and whose length preserves the finitely additive $\mu$ 
measure on $E_{\mathbf{k}}^{(j)}$ and such that if $x_{i_{1}}^{j_{1}}=x_{i_{2}}^{j_{2}}$ then $T_{k}\left(E_{i_{1}}^{\left(j_{1}\right)}\right)$ and $T_{k}\left(E_{t_{2}}^{\left(j_{2}\right)}\right)$, $k \geqq \max \left(i_{1}, i_{2}\right)$, have the same left-hand end points. Let $T_{0}\left[E_{0}^{(1)}\right]$ $=[0,1)$ and suppose $T_{i}$ have been defined having the above properties for $i=0,1, \cdots, k-1$. Let $E_{k}^{\left(j_{i}\right)}\left(i=0,1,2, \cdots, n_{j}^{\prime}\right)$ be the sets of $E_{k}^{(1)}$ contained in $E_{k-1}^{(j)}$ ordered such that $E_{k}^{\left(j_{0}\right)}=0$ and $x_{k}^{\left(j_{1}\right)}=x_{k-1}^{(j)}$. We define $T_{k}$ on these sets such that $T_{k}\left(E_{\mathrm{k}}^{\left(j_{i}\right)}\right)=\left[a+\sum_{t=1}^{j_{i}} \mu E_{\mathrm{k}}^{\left(j_{k}\right)}\right.$, $\left.a+\sum_{i=1}^{j_{i}} \mu E_{k}^{\left(j_{k}\right)}\right)$ where $a$ is the left-hand end point of $T_{k-1}\left[E_{k-1}^{(j)}\right]$; (by property (iii)) $E_{k}^{(t)} \neq 0$ implies $\mu E_{k}^{(t)}>0$ and hence $T_{k}\left(E_{k}^{\left(j_{i}\right)}\right)$ is a nondegenerate interval. $T_{k}$ is defined similarly on the remaining sets of $E_{k}^{(j)}$ and we see that the collection of transformations $T_{k}(k=0,1,2 \cdots)$ has the desired properties.

We denote by $D$ the set of left-hand end points of intervals of the form $T_{k}\left[E_{k}^{(j)}\right] ; D$ is then dense on $[0,1)$ by property (iv). But by the construction of $T_{k}$ and property (iv) the point $x_{k}^{(j)}$ is the unique point contained in all intervals $T_{i}\left(E_{i}^{(j)}\right)$ having the same left-hand end point as $T_{k}\left(E_{\mathrm{k}}^{(j)}\right)$. Thus we may define a 1-1 transformation $T$ such that $T\left[x_{k}^{(j)}\right]$ is the left-hand end point of $T_{k}\left[E_{k}^{(j)}\right]$, and then $T(X)=D$. Let $E_{\mathbf{k}}^{(j)}$ be any set in $\mathcal{P}$ and let $\bar{x} \in E_{\mathbf{k}}^{(j)}$; then $T(\bar{x}) \in T_{k}\left(E_{\mathbf{k}}^{(j)}\right)$. This implies (a) $m \mathrm{Cl} T\left(E_{k}^{(j)}\right) \leqq m T_{k}\left(E_{k}^{(j)}\right)=\mu E_{\mathbf{k}}^{(j)}$; whereas by the density of $D$ we have that (b) $\sum_{j=1}^{n_{k}} m \operatorname{Cl} T\left[E_{k}^{(j)}\right] \geqq 1$. Applying the finite additivity of $\mu$ on $\mathcal{P}$ we see that (c) $\sum_{j=1}^{n_{k}} \mu E_{k}^{(j)}=1$, and combining $\mathrm{a}, \mathrm{b}$, and $\mathrm{c}$ we see that $m \mathrm{Cl} T\left[E_{\mathrm{k}}^{(j)}\right]=\mu E_{k}^{(j)}$. This completes the proof since clearly $T[R(P)]=R T[P]$ and $[C, \nu]$ is the finite Caratheodory closure of $T(P)$ and hence also of $T[R(P)]$.

We may now describe the set isomorphism $\tau$ which arises rather naturally between the classes $[\mathcal{F}, m]$ and $[\mathcal{C}, \nu]$. Let $E \Delta F$ denote the symmetric difference $(E-F) \cup(F-E)$. If $\mathcal{E}$ is a subclass of $\mathcal{C}$ such that $E, F \in \mathcal{E}_{\text {implies }} \nu(E \Delta F)=0$ then $\tau(\varepsilon)$ is defined as that sub-class $\mathcal{F}^{*}$ of $\mathcal{F}$ which contains $\mathrm{Cl} E$ for some $E \in \mathcal{E}$ and satisfies $E, F \in \mathcal{F}^{*} \mathrm{im}$ plies $m(E \Delta F)=0$. We note several differences between this isomorphism and the point isomorphism described in the theorem. First the correspondence is not 1-1 but modulo sets of measure 0 . More important, however, if $E_{i}$ is a countable, disjoint collection of sets in $\mathcal{C}$ then in order that $U E_{i}$ be in $\mathcal{C}$ it is sufficient but not necessary that $\mathrm{UCl} E_{i}$ be in $\mathcal{F}$.

Some properties of general $\mathrm{f}$-a measures follow immediately from the corresponding properties for Jordan content. Thus we see that if $E$ is in $\mathcal{X}$ and $\mu E=\alpha>0$ then the range of the measure of subsets of $E$ contained in $\mathcal{X}$ is the interval $[0, \alpha] .{ }^{1}$ Any set $F$ possessing Jordan content differs from an open set by a set of measure 0 (i.e. $m(\mathrm{Cl} F$

${ }^{1}$ It follows from a result of Sobczyk and Hammer that this set is perfect [5]. 
$\left.\left.\cap \mathrm{Cl} F^{\prime}\right)=0\right)$. Hence, using the density of the partitions $\left\{E_{i}^{(j)}\right\}$, for any $E \in \mathcal{X}$ there are sets $P_{i} \in \mathcal{P}$ such that, $U P_{i} \in \mathcal{X}$ and $\mu\left(U P_{i} \Delta E\right)$ $=0$. In particular for the class $\mathscr{D}_{0}^{*}$ we see that any measurable set differs from a union of arithmetic progressions by a set of measure 0 .

If $E_{j}$ is a disjoint sequence of sets on $[0,1]$, if $S_{1}=U\left[\mathrm{Cl} E_{j} \cap \mathrm{Cl} E_{j}^{\prime}\right]$, $S_{2}=\left[x ; x \notin \mathrm{Cl} E_{j}, x \in \mathrm{ClU} E_{j}\right]$ and $S_{3}=\mathrm{ClU} E_{j} \cap \mathrm{Cl}\left(U E_{j}\right)^{\prime}$ then $m S_{1}=0$ if and only if $E_{j} \in \mathcal{F}$ for all $j, m S_{2}=0$ if and only if $m \mathrm{ClU} E_{\jmath}=\sum m \mathrm{Cl} E_{j}$ and $m S_{3}=0$ if and only if $\cup E_{j} \in \mathcal{F}$. Since $S_{1} \cup S_{2} \supset S_{3}$ and content is numerically equal to Lebesgue measure we see that if $E_{j} \in \mathcal{F}$ then a necessary and sufficient condition that $U E_{j} \in \mathcal{F}$ is that $m \mathrm{ClU} E_{j}$ $=\sum m \mathrm{Cl} E_{j}$. If this condition holds and if $K_{j} \in \mathcal{F}, K_{j} \subset E_{j}$, then $\cup K_{j} \in \mathcal{F}$ and $m \cup K_{j}=\sum m K_{j}$. To see this, let $S_{4}=\mathrm{U}\left(\mathrm{Cl} K_{j} \cap \mathrm{Cl} K_{j}^{\prime}\right)$ then $m S_{4}=0$; then $\mathrm{ClU} K_{i} \cap \mathrm{Cl}\left(\cup K_{i}\right)^{\prime}=K \subset S_{1} \cup S_{2} \cup S_{4}$ for if $x \in K$ and $x \in E_{i}$ for some $i$, then $x \in K_{i}$ implies $x \in S_{4}, x \notin K_{i}$ implies $x \in S_{1}$, whereas $x \notin E_{i}$ for any $i$ implies $x \in S_{2}$. By Theorem 1 these properties of $\mathcal{F}$ hold for general $\mathrm{f}$-a measures namely:

THEOREM 2. If $E_{j}$ is a sequence of disjoint sets in $\mathcal{X}$, then a necessary and sufficient condition that $U E_{j}$ is in $\mathcal{X}$ with $\mu\left(U E_{j}\right)=\sum \mu E_{j}$ is that $\mathrm{UCl} T\left(E_{j}\right)$ be in $\mathcal{F}$. If this condition holds and if $K_{j}$ are in $\mathcal{X}$ with $K_{j} \subset E_{j}$ then $U K_{j}$ is in $\mathcal{X}$ and $\mu\left(U K_{j}\right)=\sum \mu K_{j}$.

The last statement of the theorem may be applied to show, for example, that in $\mathscr{D}_{0}^{*}$ the set of all primes is measurable and has measure 0 , a fact noted by Buck [1]. This follows since we may find a sequence of arithmetic progressions, each containing one prime whose union is the space of all positive integers and with sum of measures 1.

\section{BIBLIOGRAPHY}

1. R. C. Buck, The measure theoretic approach to density, Amer. J. Math. vol. 68 (1946) pp. 560-580.

2. E. F. Buck and R. C. Buck, $A$ note on finitely additive measures, Amer. J. Math. vol. 69 (1947) pp. 413-420.

3. P. R. Halmos and J. von Neumann, Operator methods in classical mechanics, II, Ann. of Math. vol. 43 (1942) pp. 332-350.

4. P. R. Halmos, Measure theory, New York, 1950.

5. A. Sobczyk and P. C. Hammer, Ranges of additive set functions, Duke Math. J. vol. 11 (1944) pp. 847-851.

SYraCUSE UNIVERSITY 